\title{
Reprogram Frozen Embryo Transfers to Allow For a More "Natural" Approach
}

\author{
Bruce Pier ${ }^{1}$, Logan Havemann ${ }^{1}$, and Ryan Heitmann ${ }^{2}$ \\ ${ }^{1}$ Madigan Army Medical Center \\ ${ }^{2}$ West Virginia University Center for Reproductive Medicine
}

January 14, 2021

Reprogram Frozen Embryo Transfers to Allow For a More "Natural" Approach

Bruce D. Pier, MD ${ }^{1}$ Logan M. Havemann, MD ${ }^{1}$ Ryan J. Heitmann, DO ${ }^{1,2}$

${ }^{1}$ Division of Reproductive Medicine, Madigan Army Medical Center, Tacoma, WA ${ }^{2}$ Center for Reproductive Medicine, West Virginia University, Morgantown, WV

Corresponding Author:Bruce D. Pier, MD Division of Reproductive Endocrinology and Infertility Department of Obstetrics and Gynecology Madigan Army Medical Center Tacoma, WA 98431 email: bruce.d.pier3.mil@mail.mil telephone: 253-968-3276

Shortened Running Title: Reprogram the Frozen Embryo Transfer

The views expressed in this manuscript are those of the authors and do not reflect the official policy or position of the Department of the Army, Department of Defense, or the United States Government.

Many advances have been made in the area of assisted reproductive technologies (ART) over the last 40 years. Ultimately, the cumulative success of these advancements comes down to the interaction of a competent embryo and a receptive uterine endometrium during the optimal window of implantation. Advancements in cryopreservation techniques has allowed for the separation of the in-vitro fertilization process into oocyte retrieval with cryopreservation of either oocytes or embryos followed by the embryo transfer at a to-bedetermined time in the future (termed "freeze-all"). Common reasons for the increased use of freeze-all strategies include, but are not limited to, use in PGT-A, risk reduction for those at high risk of OHSS due to hyper-response, elevated progesterone on the day of oocyte maturation trigger, identification of uterine (polyps, fibroids, adenomyosis) or tubal (hydrosalpinx) anomalies, and random start ovarian stimulation, most commonly done for fertility preservation in newly diagnosed cancer patients. ${ }^{1-3}$

Adverse pregnancy outcomes have been noted with both fresh and frozen (FET) embryo transfer cycles. ${ }^{4}$ Determining the optimal treatment paradigm, fresh or frozen transfer, to optimize live birth/delivery outcomes is still a highly debated topic . Outcome differences evaluating different FET endometrial preparation protocols is one area predominately studied via retrospective data. ${ }^{1}$ Herein, we discuss both hormonal or programmed along with natural FET cycles and offer a call for high quality randomized trials to help advance our knowledge of this complex topic.

The number of ART cycles have increased globally over the last decade, with over 1,200,000 cycles performed in the United States and Europe combined in 2016. ${ }^{5,6}$ The use of frozen embryo transfer has seen a drastic increase during this time, with some estimates suggesting a tripling in women over 40 in the United States between 1996-2013. ${ }^{7}$ As the increase in FET cycles has taken place, studies initially demonstrated a lower incidence of small for gestational age (SGA) infants born after a frozen embryo transfer. This represents a very 
attractive finding given data demonstrating increased SGA seen in fresh embryo transfer cycles. This finding, however, has been tempered in recent years, as data now suggests increasing rates of maternal hypertensive disorders of pregnancy (mHTN) and infants born large for gestational age (LGA) following frozen embryo transfer. ${ }^{4}$ A recent review from our group reviewed the possible pathological rationale for these findings, with perhaps the most compelling evidence suggesting the loss of the corpus lutuem in programmed frozen embryo transfer cycles as the leading cause of the association between FET and LGA/mHTN disorders. ${ }^{8}$

Frozen embryo transfers are performed three to five days post-ovulation during the natural menstrual cycle (deemed a natural transfer), or with a programmed menstrual cycle using exogenous estradiol and progesterone supplementation. Programmed FET cycles are especially useful for patients with irregular menses, but are used in all patient types given the ease of scheduling of transfers juxtaposed with the possible unpredictable nature of scheduling natural cycle FETs. A recent study by Alur-Gupta el al, demonstrated similar live birth rates after programmed or natural FET cycles. However, only $10 \%$ of the 1028 transfers in this study were done with a natural cycle preparation. ${ }^{9}$ A recent retrospective study from Sweden investigated differences in obstetrical outcomes with programmed cycles without a corpus luteum, compared to natural or modified FET cycles. Natural cycles represented over $60 \%$ of all cycles evaluated and demonstrated that programmed FET cycles were associated with increased rates of mHTN, cesarean section, post-partum hemorrhage, post-term birth and macrosomia when compared to natural/modified FET. The difference in postpartum hemorrhage (PPH) was particularly striking, with $19.4 \%$ of patients in the programmed cycles experiencing PPH compared to $7.9 \%$ in the natural cycle cohort. ${ }^{10}$ Similarly, a recent study by Makhijani et al, demonstrated a 2-fold increase in mHTN in programmed cycles compared to natural cycles, and concluded that natural cycle FET should be the first-line option given to patients undergoing FET. ${ }^{11}$

What leads to the differences observed? Research has also been conducted to provide pathophysiologic rationale to this association. A recent review by Singh et al discussed the role of the corpus luteum in early pregnancy, concluding programmed cycles and subsequent lack of a corpus luteum, suppress the production of vasoactive compounds which may increase the risk of pre-eclampsia. Specifically, the absence of circulating relaxin and vascular endothelial growth factor in early pregnancy likely contributes to abnormal placentation during programmed FET cycles, which may represent the cause for increased obstetrical complications observed after these cycles. ${ }^{12}$

It is unclear from published data the true prevalence of programmed FET cycle use in the United States, but anecdotally, our experience is the use of programmed cycles is similar to the data presented by Alu-Gupta el al. ${ }^{9}$ While research to date does not demonstrate a definitive relationship between programmed FET and LGA/mHTN, the literature seems to present a strong enough cause for our community to rethink the high use of programmed FET cycles. We present the need for more high quality research, particularly with randomized controlled trials, to substantiate these findings before completely abandoning the programmed cycle. Even so, given the possible decrease in LGA/mHTN pregnancy complications, it would seem beneficial for infertility clinics across the world to strongly considering using natural FET cycles over programmed cycles whenever possible.

\section{Disclosure of interests}

None declared. Completed disclosure of interests forms are available to view online as supporting information.

\section{Contribution to authorship}

All authors contributed to the creation of the draft of this commentary, reviewed the final draft for accuracy and provided comments.

\section{Details of ethics approval}

This work is a commentary on the state of the literature and is not a scientific study in itself. Institutional Review Board approval was not required from any of the authors' institutions.

\section{Funding}


There was no funding for this work.

\section{References}

1. Celada P and Bosch E. Freeze-all, for whom, when, and how. Ups J Med Sci. 2020 May;125(2):104-111.

2. Healy MW, Patounakis G, Connell MT, Devine K, DeCherney AH, Levy MJ, Hill MJ. Does a frozen embryo transfer ameliorate the effect of elevated progesterone seen in fresh transfer cycles? Fertil Steril. 2016 Jan;105(1):93-9.e1. doi:

3. Chen ZJ, Shi Y, Sun Y, Zhang B, Liang X, Cao Y, Yang J, Liu J, Wei D, Weng N, Tian L, Hao C, Yang D, Zhou F, Shi J, Xu Y, Li J, Yan J, Qin Y, Zhao H, Zhang H, Legro RS. Fresh versus Frozen Embryos for Infertility in the Polycystic Ovary Syndrome. N Engl J Med. 2016 Aug 11;375(6):523-33.

4. Maheshwari A, Pandey S, Raja EA, Shetty A, Hamilton M, Bhattacharya S. Is Frozen Embryo Transfer Better for Mothers and Babies? Can Cumulative Meta-Analysis Provide a Definitive Answer? Hum Reprod Update. 2018; 24:35-58.

5. Sunderam S, Kissin DM, Zhang Y, Folger SG, Boulet SL, Warner L, Callaghan WM, Barfield WD. Assisted Reproductive Technology Surveillance - United States, 2016. MMWR Surveill Summ. 2019;68(No. SS4):1-23.

6. European IVF-monitoring Consortium (EIM)++ for the European Society of Human Reproduction and Embryology (ESHRE), Wyns C, Bergh C, Calhaz-Jorge C, De Geyter C, Kupka MS, Motrenko T, Rugescu I, Smeenk J, Tandler-Schneider A, Vidakovic S, Goossens V. ART in Europe, 2016: results generated from European registries by ESHRE. Hum Reprod Open. 2020 Jul 31;2020(3):hoaa032.

7. Hipp H, Crawford S, Kawwass JF, Boulet SL, Grainger DA, Kissin DM, Jamieson D. National trends and outcomes of autologous in vitro fertilization cycles among women ages 40 years and older. J Assist Reprod Genet. 2017 Jul;34(7):885-894. 8.

8. Gaume R, Heitmann RJ, Luizzi J, Pier BD. Large For Gestational Age Following Frozen Embryo Transfer: An Evaluation of the Possible Causes for this Relationship. F\&S Reviews. 2020, doi:http://doi.org/10.1016/j.xfnr.2020.10.002

9. Alur-Gupta S, Hopeman M, Berger DS, Gracia C, Barnhart KT, Coutifaris C, Senapati S. Impact of method of endometrial preparation for frozen blastocyst transfer on pregnancy outcome: a retrospective cohort study. Fertil Steril. 2018 Sep;110(4):680-686.

10. Ginström Ernstad E, Wennerholm UB, Khatibi A, Petzold M, Bergh C . Neonatal and maternal outcome after frozen embryo transfer: Increased risks in programmed cycles. Am J Obstet Gynecol. 2019;221:126e1-18

11. Makhijani R, Bartels C, Godiwala P, Bartolucci A, Nulsen J, Grow D, Benadiva C, Engmann L. Maternal and perinatal outcomes in programmed versus natural vitrified-warmed blastocyst transfer cycles. Reprod Biomed Online. 2020 Aug;41(2):300-308.

12. Singh B, Reschke L, Segars J, Baker VL. Frozen-thawed embryo transfer: the potential importance of the corpus luteum in preventing obstetrical complications. Fertil Steril. 2020; 113:252-59. 